\title{
The Computer Model and the Algorithm of Designing \\ a Target Environment of Remote Monitoring Areas \\ Using Thermal Tomograms with Account \\ of their Geographical Location \\ and Meteorological Conditions
}

\author{
Igor N. Ishchuk ${ }^{\mathrm{b}}$ and Alexey A. Dolgov** \\ ${ }^{a}$ Military Education and Research Centre of Military-Air Forces \\ «Military-Air Academy \\ Named After Professor N.E. Zhukovsky and Yu.A. Gagarin» \\ Voronezh, Russian Federation \\ ${ }^{b}$ Siberian Federal University \\ Krasnoyarsk, Russian Federation
}

Received 30.09.2019, received in revised form 10.12.2019, accepted 21.01.2020

Abstract. The article considers a computer model and an algorithm used for designing a target environment based on extrapolation of temperature fields of remote monitoring areas according to databases of thermal tomograms of analog objects, underlying surfaces, geographical location and meteorological conditions. A mathematical statement of the problem, concerning simulating the temperature fields of "analogue objects" and bottoming surfaces of remote monitoring areas by means of their thermal tomograms, is described. The outcomes of computer simulation of the spatial distribution of temperature fields of the remote monitoring area based on the results of the field experiment are presented.

Keywords: computer simulation, a mathematical model, target environment, an analogue object, remote monitoring, unmanned aerial vehicle.

Citation: Ishchuk I.N., Dolgov A.A. The computer model and the algorithm of designing a target environment of remote monitoring areas using thermal tomograms with account of their geographical location and meteorological conditions, J. Sib. Fed. Univ. Eng. \& Technol., 2020, 13(3), 350-360. DOI: 10.17516/1999-494X-0226

(C) Siberian Federal University. All rights reserved

This work is licensed under a Creative Commons Attribution-Non Commercial 4.0 International License (CC BY-NC 4.0).

* Corresponding author E-mail address: alexdolgov88-08@rambler.ru 


\title{
Компьютерная модель и алгоритм построения фоноцелевой обстановки районов
}

\section{Дистанционного мониторинга по тепловым томограммам с учетом их географического положения \\ и метеорологических условий}

\author{
И.Н. Ищукб, А.А. Долгов \\ ${ }^{a}$ Военный учебно-научный изентр Военно-воздушных сил \\ «Военно-воздушная академия \\ имени профессора Н.Е. Жуковского и Ю.А. Гагарина» \\ Российская Федерация, Воронеж \\ ${ }^{\sigma}$ Сибирский федеральный университет \\ Российская Федераџия, Красноярск
}

\begin{abstract}
Аннотация. В статье рассматриваются компьютерная модель и алгоритм построения фоноцелевой обстановки на основе экстраполяции температурных полей районов дистанционного мониторинга по базам данных тепловых томограмм объектов-аналогов, подстилающих поверхностей, географического положения и метеорологических условий. Представлена математическая постановка задачи моделирования температурных полей «объектов-аналогов» и подстилающих поверхностей районов дистанционного мониторинга по их тепловым томограммам. Приведены результаты компьютерного моделирования пространственного распределения температурных полей района дистанционного мониторинга по результатам натурного эксперимента.
\end{abstract}

Ключевые слова: компьютерное моделирование, математическая модель, фоноцелевая обстановка, объект-аналог, дистанционный мониторинг, беспилотный летательный аппарат.

Цитирование: Ищук, И.Н. Компьютерная модель и алгоритм построения фоноцелевой обстановки районов дистанционногомониторингапо тепловым томограммамс учетомих географическогоположения иметеорологических условий / И.Н. Ищук, А.А. Долгов // Журн. Сиб. федер. ун-та. Техника и технологии, 2020. 13(3). С. 350-360. DOI: $10.17516 / 1999-494 X-0226$

\section{Введение}

В настоящее время в области разработки беспилотных летательных аппаратов (БпЛА) все больший интерес приобретают вопросы создания полностью автономных комплексов, способных выполнять задачи в условиях отсутствия связи с пунктом управления и спутниковыми навигационными системами. Одной из главных проблем при создании подобных комплексов с БпЛА является ориентация в пространстве, так как выполнение задач дистанционного мониторинга (ДМ) привязано к конкретному участку местности.

Для решения вышеуказанных задач комплексу с БпЛА требуется «эталонный» массив данных, с которым он будет сопоставлять данные, получаемые с его оптико-электронной системы (ОЭС). Одними из перспективных направлений решения вышеназванной проблемы может стать использование в качестве нового информационного слоя геоинформационных систем земной поверхности тепловых томограмм эталонных «объектов-аналогов» и подстилающих поверхностей и построение на их основе пространственного распределения температурных 
полей районов ДМ с учетом их географического местоположения, времени суток и метеорологических условий.

\section{1. Постановка задачи}

Рассмотрим район ведения ДМ с расположенными на нем надповерхностными (НПО) и подповерхностными объектами (ППО), а также тепловые потоки, действующие в нем (рис. 1).

Распространение температурных полей на поверхности и вглубь профиля района ДМ, представленного на рис. 1, может быть описано с помощью многослойных тепловых моделей для анизотропных негомогенных сред: однослойной - для фона, двухслойной - для надповерхностного объекта, расположенного на земной поверхности, например на грунте; трехслойной - для подповерхностного объекта, расположенного в грунте в приповерхностном слое (рис. 2).

Пространственное распределение температурных полей в многослойной полуограниченной анизотропной среде с учетом граничных условий 2-го рода теплового баланса земной поверхности (ГУ ІІ) и граничных условий сопряжения слоистых сред, учитывающего неидеальность теплового контакта граничащих поверхностей (ГУ IV), можно описать следующим образом [1-3]:

$$
\left\{\begin{array}{l}
C_{\rho_{j}}(\theta) \frac{\partial T_{j}}{\partial \tau}=\frac{\partial}{\partial x}\left(\left[\lambda_{j}(\theta)\right]_{11} \frac{\partial T_{j}}{\partial x}\right)+2 \frac{\partial}{\partial x}\left(\left[\lambda_{j}(\theta)\right]_{12} \frac{\partial T_{j}}{\partial y}\right)+2 \frac{\partial}{\partial x}\left(\left[\lambda_{j}(\theta)\right]_{13} \frac{\partial T_{j}}{\partial z}\right)+ \\
+\frac{\partial}{\partial y}\left(\left[\lambda_{j}(\theta)\right]_{22} \frac{\partial T_{j}}{\partial y}\right)+2 \frac{\partial}{\partial y}\left(\left[\lambda_{j}(\theta)\right]_{23} \frac{\partial T_{j}}{\partial z}\right)+\frac{\partial}{\partial z}\left(\left[\lambda_{j}(\theta)\right]_{33} \frac{\partial T_{j}}{\partial z}\right)-C_{w_{j}} \frac{\partial T_{j}}{\partial y} \\
(1-V) Q-B_{0}^{*}=-\left.\lambda(\theta) \frac{\partial T_{0}}{\partial z}\right|_{z=0}+\left.C^{*} \rho^{*} k \frac{\partial T_{0}}{\partial z}\right|_{z=0}+\left.L_{\rho} k \frac{\partial s}{\partial z}\right|_{z=0} ; \\
\left.\lambda_{j}(T, \theta) \frac{\partial T_{j}}{\partial z}\right|_{z=h_{j}}=\frac{1}{R_{T}}\left(\left.T_{j+1}\right|_{z=+\Delta h_{j}}-\left.T_{j}\right|_{z=-\Delta h_{j}}\right) ; \\
\left.\lambda_{j}(T, \theta) \frac{\partial T_{j}}{\partial z}\right|_{z=-\Delta h_{j}}=\left.\lambda_{j+1}(T, \theta) \frac{\partial T_{j+1}}{\partial z}\right|_{z=+\Delta h_{j}} ; \\
\left.T_{N}\right|_{z=H}=\varphi_{1}(x, y, H) ; \\
\left.T_{0}\right|_{z=0}=\varphi_{0}(x, y, 0)
\end{array}\right.
$$

где $T=T(x, y, z, \tau)$ - неизвестная функция температуры, $\tau \in\left[\tau_{0}, \tau_{1}\right],(x, y, z) \in D=\Omega \times[0, H], \Omega \subset R^{2}$, $\Omega$ - поверхность полуограниченной среды; $C_{p}, C_{w}-$ теплоемкости пористой среды и жидкости соответственно; $\left[\lambda_{j}(\theta)\right]_{11},\left[\lambda_{j}(\theta)\right]_{12},\left[\lambda_{j}(\theta)\right]_{13},\left[\lambda_{j}(\theta)\right]_{22},\left[\lambda_{j}(\theta)\right]_{23},\left[\lambda_{j}(\theta)\right]_{33}-$ компоненты тензора эффективной теплопроводности негомогенной пористой среды (почвы) с разрывными коэффициентами $(j \in[0, H])$ в зависимости от потенциальной температуры $\theta$, где $C^{*}$ - удельная изобарная теплоемкость воздуха, $\rho$ - плотность воздуха, $k$ - коэффициент турбулентности; $s$ - массовая доля водяного пара, $L$ - удельная теплота парообразования; $B_{0}^{*}=\delta\left(\sigma T_{0}^{4}-\delta_{a} \sigma T_{\Pi}^{4}\right)$ - эффективное излучение верхнего слоя поверхности, $\delta$ - поглощательная способность верхнего слоя поверхности, $\sigma$ - постоянная Стефана - Больцмана; $q$ - плотность теплового потока, $C_{v}-$ удельная теплоемкость водяного пара, $R_{T}$ - коэффициент термического сопротивления, $H$ - координата нижней границы, $\varphi_{0}(x, y), \varphi_{1}(x, y)$ - известные функции начального распределения температур; $Q=Q(x, y, z, \tau)$ поток суммарной солнечной радиации [2]. 


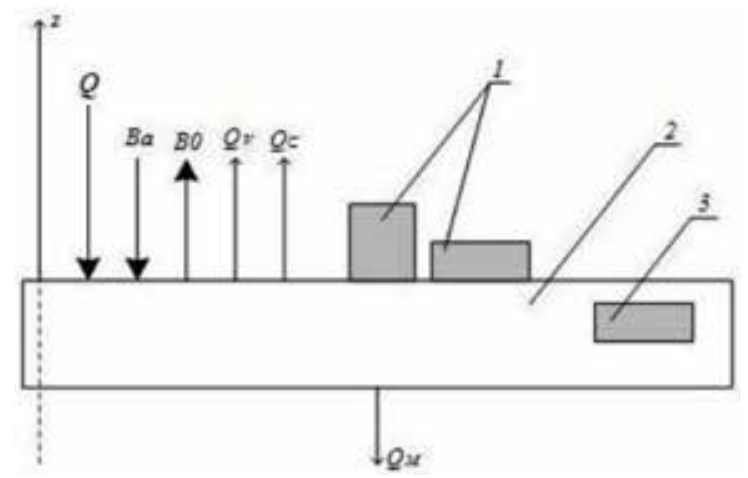

Рис. 1. Упрощенная модель района ведения дистанционного мониторинга: 1 - надповерхностный объект; 2 - грунт (фон); 3 - подповерхностный объект

Fig. 1. Simplified model of remote monitoring area: 1 - surface object; 2 - ground (background); 3 - subsurface object

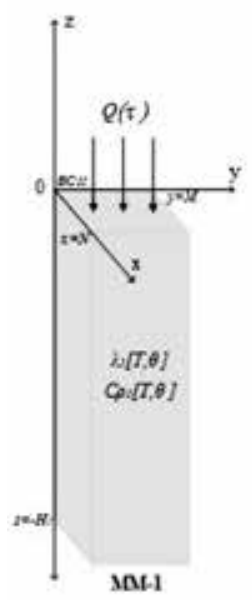

1)

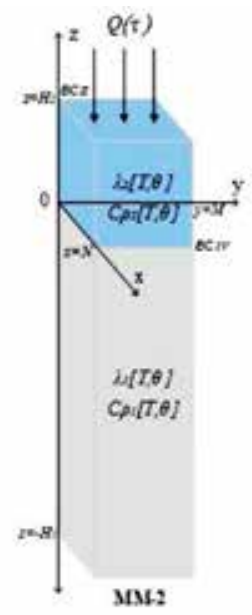

2)

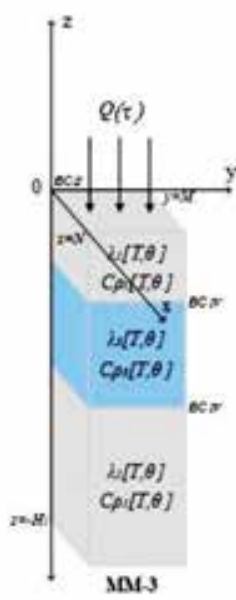

3)

Рис. 2. Многослойные тепловые модели анизотропных негомогенных сред: 1 - однослойная (фон); 2 двухслойная (НПО/фон); 3 - трехслойная (фон/ППО/фон)

Fig. 2. Multilayer thermal models of anisotropic inhomogeneous media: 1 - single-layer (background); 2 - twolayer (overground object / background); 3 - three-layer (background / underground object/background)

\section{2. Алгоритм моделирования}

Рассмотрим алгоритм моделирования пространственного распределения нестационарных температурных полей поверхности района ДМ по его тепловым томограммам путем численного решения параболического дифференциального уравнения теплопроводности в частных производных (1), учитывающего граничные условия теплового баланса земной поверхности и граничные условия сопряжения слоистых сред на основе метода конечных элементов (рис. 3).

Опишем данный алгоритм.

Шаг 1. Задание исходных данных:

- пространственное распределение теплофизических полей (ТФП) (теплопроводность $\lambda$; удельная теплоемкость $C$, плотность вещества $\rho$ ); 


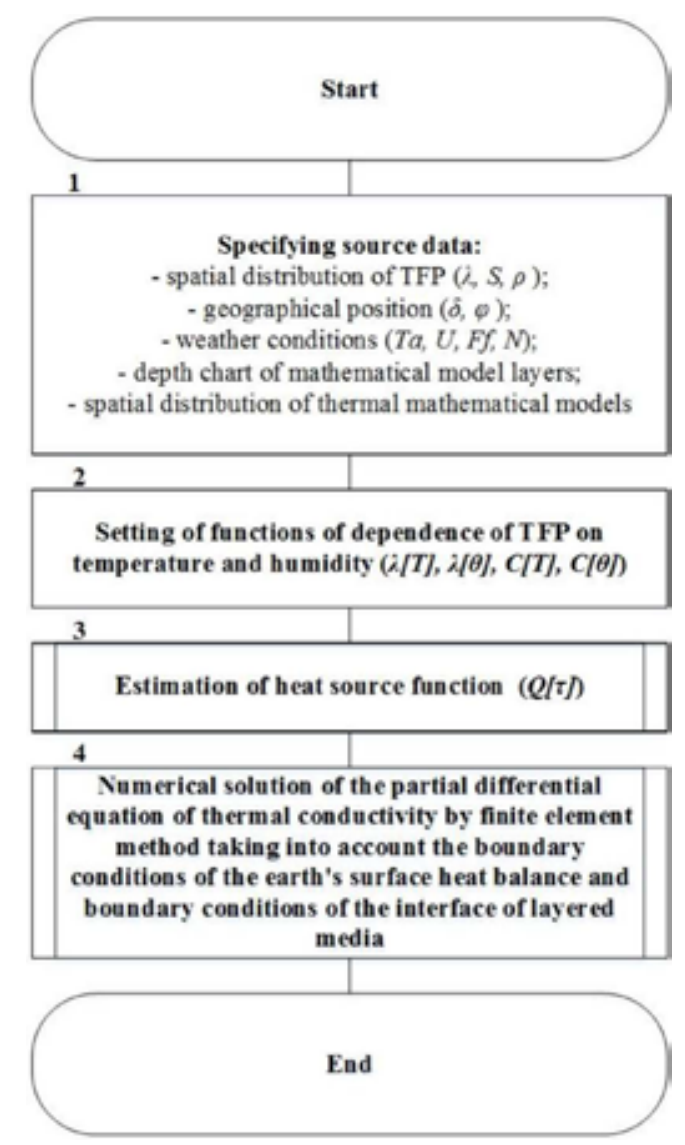

Рис. 3. Алгоритм моделирования температурных полей района дистанционного мониторинга по тепловой томограмме с учетом его географического положения и метеорологических условий

Fig. 3. Algorithm for modeling the temperature fields of the remote monitoring area by thermal tomogram, taking into account its geographical location and meteorological conditions

- параметры географического положения (угол склонения Солнца $\delta$; широта места наблюдения $\varphi$ );

- метеорологические условия (температура воздуха в приземном слое $T_{a}$; относительная влажность воздуха $U$; скорость ветра в приземном слое $F_{f}$; облачность $N$ );

- глубинограмма слоев математической модели;

- пространственное распределение тепловых математических моделей анизотропных негомогенных сред.

Шаг 2. Задание функций зависимости ТФП от эффективной температуры и влажности среды.

Аппроксимация распределения ТФП с помощью эмпирических зависимостей:

$$
\begin{gathered}
\lambda_{i}(T)=\lambda_{0 i}\left[1+a_{i}\left(T-T_{0}\right)\right], \quad i=1 . \mathrm{n} \\
\lambda_{i}(\theta)=a_{0 i}+a_{1 i} \theta+a_{2 i} \theta^{1 / 2}, \quad i=1 . \mathrm{n} \\
\mathrm{C}_{i}(T)=b_{0 i}+b_{1 i} T+b_{2 i} T^{2}+b_{-2} T^{-2}, \quad i=1 . \mathrm{n} \\
-354-
\end{gathered}
$$


где $a, a_{0}, a_{1}, a_{2}, b_{0}, b_{1}, b_{2}, b_{-2}$ - эмпирические коэффициенты, зависящие от природы вещества; $n=3$ - количество слоев [4].

Шаг 3. Оценка функции источника тепла.

Распределение теплового потока на поверхности района ДМ можно оценить следующим образом:

$$
\left\{\begin{array}{l}
Q^{*}=F_{0}^{*} \int_{-t_{0}}^{t_{0}}\left(\sin \varphi \sin \delta+\cos \varphi \cos \delta \sin \frac{2 \pi}{P} t\right) \frac{d t}{R^{2}} \\
Q^{0}=Q^{*} p_{\lambda}^{m} \\
m=\left\{\begin{array}{l}
\frac{1}{\sin (h)}=\operatorname{cosec}(h), \quad h \geq 30^{\circ} \\
\frac{53}{h}, \quad h \leq 30^{0}
\end{array}\right. \\
Q=Q^{0}(1-(a+b n) n)
\end{array}\right.
$$

где $Q^{*}-$ поток солнечной радиации, приходящий на верхнюю границу атмосферы; $F_{0}=1.88-$ $1.98 \kappa a{ }^{*} \mathrm{~cm}^{2} /$ мин - астрономическая солнечная постоянная; $\pm t_{0}-$ время восхода и захода Солнца, отсчитывая от полудня; $R=r / r_{0}$ - отношение произвольного и среднего расстояний от Земли до Солнца; $\varphi$ - широта места наблюдения; $\delta$ - угол склонения Солнца; $P$ - период вращения Земли; $\frac{2 \pi}{P} t$ - часовой угол; $t$ - время, отсчитываемое от полудня [2]; $Q^{\circ}-$ поток суммарной солнечной радиации, приходящий на поверхность при безоблачном небе; $p_{\lambda}=\exp \left(-\alpha_{\lambda}\right)-\operatorname{cpeд-~}$ ний коэффициент прозрачности атмосферы при безоблачном небе; $\alpha_{\lambda} \approx 0.2-$ средний коэффициент ослабления солнечной радиации в атмосфере; $m$ - оптическая масса атмосферы; $h$ - высота подъема Солнца; $Q$ - поток суммарной солнечной радиации, приходящий на поверхность, ослабленный облачностью; $n$ - общая облачность (в долях единицы); $\alpha, b$ - эмпирические коэффициенты, зависящие от географической широты места наблюдения (а $0.4 ; a \simeq 0.4 ; b \simeq 0.38$ для $\left.\varphi=50^{\circ}\right)[3]$.

Распределение потока суммарной солнечной радиации в течение времени ДМ опишем с помощью выражения

$$
\Delta Q_{j}=Q\left(\cos \left(\frac{2 \gamma \Delta \tau_{j}}{\tau}\right)-\cos \left(\frac{2 \gamma \Delta \tau_{j-1}}{\tau}\right)\right)
$$

где $\lambda$ - максимальный угол подъема Солнца над горизонтом в течение суток; $\tau$ - продолжительность дня; $\left[\Delta \tau_{j-1}, \Delta \tau_{j}\right]$ - временной интервал распределения солнечной радиации $\Delta Q_{j}, j \in[1, \tau]-$ количество временных отсчетов от восхода Солнца [2, 3].

При этом введем следующее допущение: тепловой поток распределяется по всей площади равномерно, но с разной плотностью в течение времени ДМ. При наличии актинометрических измерений, полученных с помощью пиранометра и балансомера, необходимость в аппроксимации функции плотности теплового потока отпадает.

Шаг 4. Численное решение дифференциального уравнения теплопроводности в частных производных методом конечных элементов с учетом ГУ II - теплового баланса земной поверхности и ГУ IV - сопряжения слоистых сред (1).

\section{3. Экспериментальная апробация}

Для экспериментальной апробации представленного выше алгоритма был выбран участок местности с расположенными на нем ложными объектами техники - «объектами-аналогами»:

$$
-355-
$$


макет БМП и макет танка под маскировочным комплектом МКТ-2 (рис. 4). Место проведения эксперимента: г. Воронеж (широта места 51² 40’ с.ш.), дата проведения: 13-14 октября 2018 года. Съемка производилась: в видимом диапазоне с использованием БпЛА DJI Phantom 4 с установленной на нем штатной ОЭС, в инфракрасном (ИК) диапазоне - ОЭС БпЛА «Гранат-2», установленной на БпЛА мультироторного типа с высоты 200 м. Численный расчет температурных полей представленного на рис. 4 района ДМ производился по его тепловым томограммам (рис. 5) с использованием специального программного обеспечения, разработанного в среде

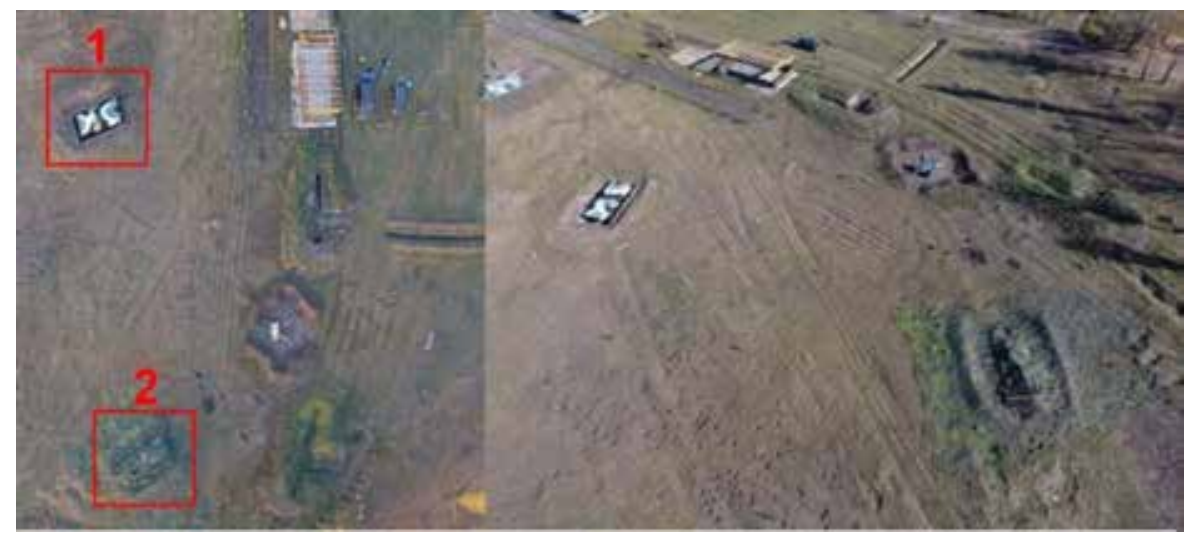

Рис. 4. Район дистанционного мониторинга с ложными объектами: 1 - макет БМП; 2 - макет танка под маскировочным комплектом МКТ-2

Fig. 4. Remote monitoring area with false objects: 1 - BMP layout; 2 - layout of the tank under the camouflage kit MKT-2
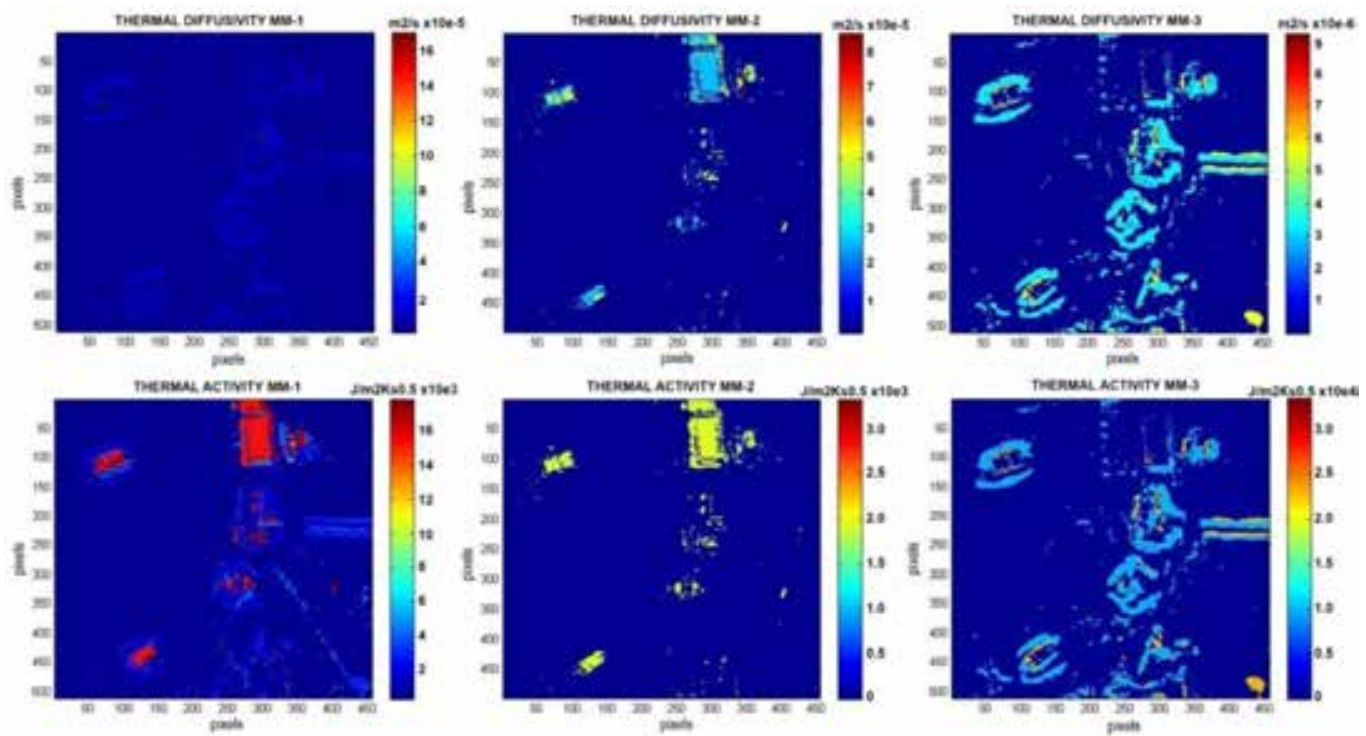

Рис. 5. Распределение теплофизических полей района дистанционного мониторинга: верхний ряд - по температуропроводности; нижний - по тепловой активности

Fig. 5. Distribution of thermal fields in the area of remote monitoring: top row on thermal diffusivity; bottom thermal activity 
Таблица 1. Астрономические и метеорологические условия

Table 1. Astronomical and meteorological conditions

\begin{tabular}{|c|c|c|c|c|}
\hline \multirow{2}{*}{ Дата, время } & \multicolumn{2}{|c|}{ Широта места: 5140’ с.ш. } & \multicolumn{2}{c|}{ Склонение Солнца: $-7.415^{\circ}$} \\
\cline { 2 - 5 } & $\begin{array}{c}\text { Температура } \\
\text { воздуха } \mathrm{T}_{\mathrm{A}}{ }^{\circ} \mathrm{C}\end{array}$ & $\begin{array}{c}\text { Относительная } \\
\text { влажность воздуха } \\
\mathrm{U}, \%\end{array}$ & $\begin{array}{c}\text { Скорость ветра, } \\
\text { м/с }\end{array}$ & Общая облачность \\
\hline 13.10 .1812 .00 & 16.1 & 51 & 6 & $2-3$ \\
\hline 13.10 .1815 .00 & 18.6 & 37 & 5 & $7-8$ \\
\hline 13.10 .1818 .00 & 16.0 & 44 & 3 & $0-1$ \\
\hline 13.10 .1821 .00 & 13.1 & 51 & 4 & $0-1$ \\
\hline 14.10 .1800 .00 & 10.8 & 61 & 4 & $0-1$ \\
\hline 14.10 .1803 .00 & 9.0 & 67 & 2 & $2-3$ \\
\hline 14.10 .1806 .00 & 7.2 & 76 & 3 & 0 \\
\hline
\end{tabular}

MATLAB с подключением программного пакета моделирования тепловлагопереноса в пористых неоднородных средах HYDRUS-1D.

Тепловая томограмма района ДМ была получена в результате решения коэффициентной обратной задачи теплопроводности путем совместной обработки многоспектральных изображений в видимом и ИК-диапазонах, сформированных в кубоид изображений $[3,5]$.

Астрономические и метеорологические условия в районе проведения эксперимента были получены с метеостанции, расположенной в аэропорту Воронеж Чертовицкое и представлены в табл. 1 [6].

Экспериментальные распределения термодинамических температурных полей района ДМ были рассчитаны по методике, описанной в [7] с использованием эталонных материалов. Результаты компьютерного моделирования распределения температурных полей по тепловым томограммам представлены на рис. 6.

Для проверки адекватности применяемых компьютерной модели и алгоритма построения температурных полей покажем значения усредненной невязки по абсолютным значениям температур на объектах, указанных на рис. 4 , и относительную погрешность ошибки на всем временном интервале моделирования (рис. 7, 8).

После анализа полученных зависимостей видно, что для рассматриваемых объектов величина относительной погрешности при моделировании их температурных полей на всем протяжении времени моделирования не превышает 4.5 - $5 \%$, что может свидетельствовать об адекватности применяемых компьютерной модели и алгоритма построения фоноцелевой обстановки районов дистанционного мониторинга.

\section{Вывод}

Использование предложенной компьютерной модели и алгоритма построения фоноцелевой обстановки районов дистанционного мониторинга по тепловым томограммам с учетом их географического положения и метеорологических условий позволяет следующее:

$$
-357-
$$



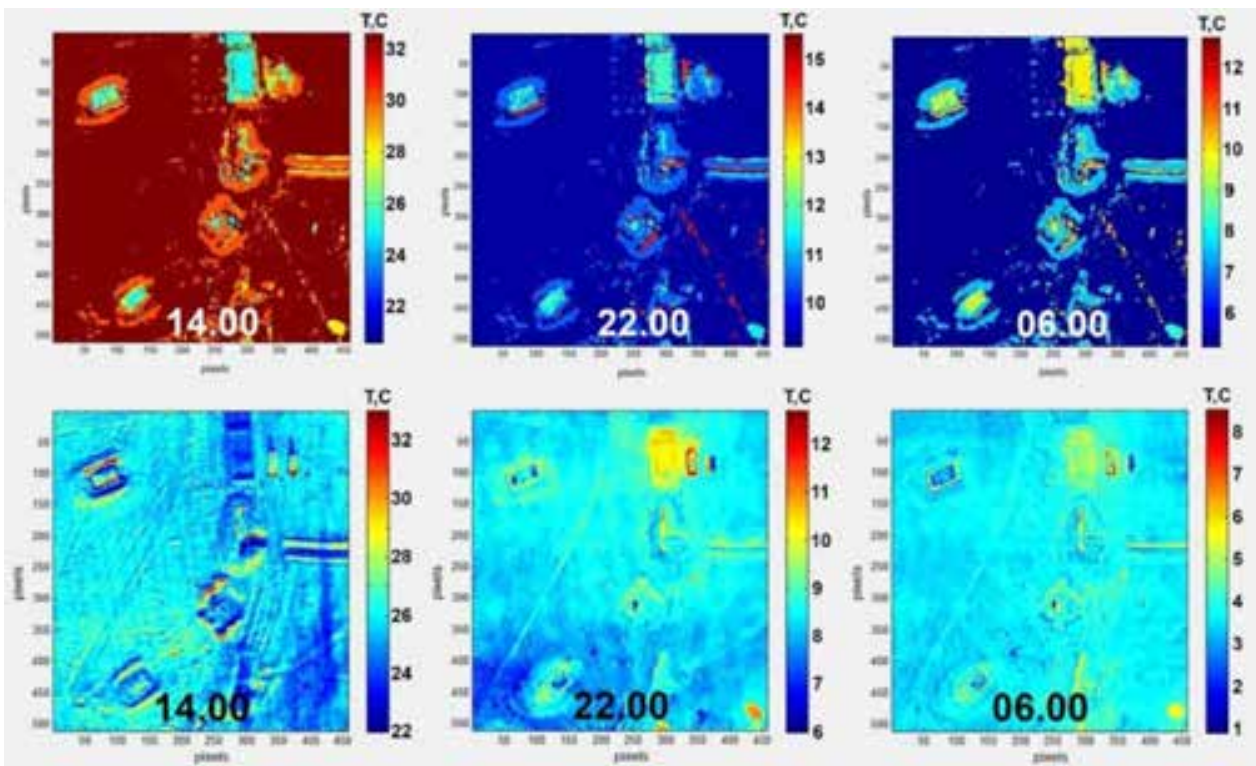

Рис. 6. Пространственное распределение температурных полей района дистанционного мониторинга: верхний ряд - компьютерное моделирование; нижний ряд - эксперимент

Fig. 6. Spatial distribution of temperature fields of remote monitoring area: upper row-computer modeling; lower row - experiment
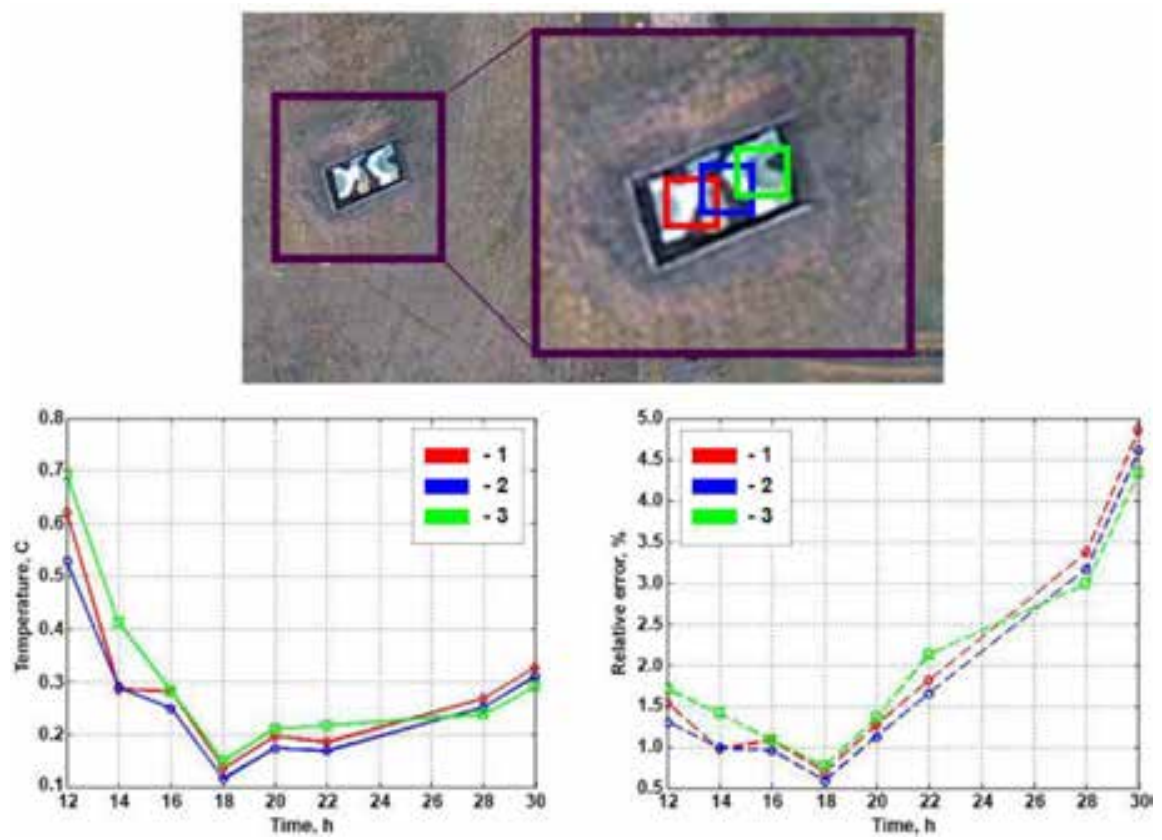

Рис. 7. Динамика изменения усредненной невязки по температуре (слева) и относительной погрешности (справа) моделирования температурных полей объекта «макет БМП» во времени: 1 - пиксель с координатами $\mathrm{x}=71 ; \mathrm{y}=112 ; 2-$ пиксель с координатами $\mathrm{x}=90 ; \mathrm{y}=99 ; 3-$ пиксель с координатами $\mathrm{x}=95$; $\mathrm{y}=87$; площадь участка для всех точек $\Delta \mathrm{x}=15$ пикс.; $\Delta \mathrm{y}=15$ пикс.

Fig. 7. Dynamics of changes in the average residual temperature (left) and relative error (right) modeling temperature fields object "layout BMP" in time: 1 - pixel coordinates $\mathrm{x}=71 ; \mathrm{y}=112 ; 2$ - pixel coordinates $\mathrm{x}=90 ; \mathrm{y}=99$; 3 - pixel coordinates $\mathrm{x}=95 ; \mathrm{y}=87$; plot area for all points $\Delta \mathrm{x}=15$ pixels; $\Delta \mathrm{y}=15$ pixels 


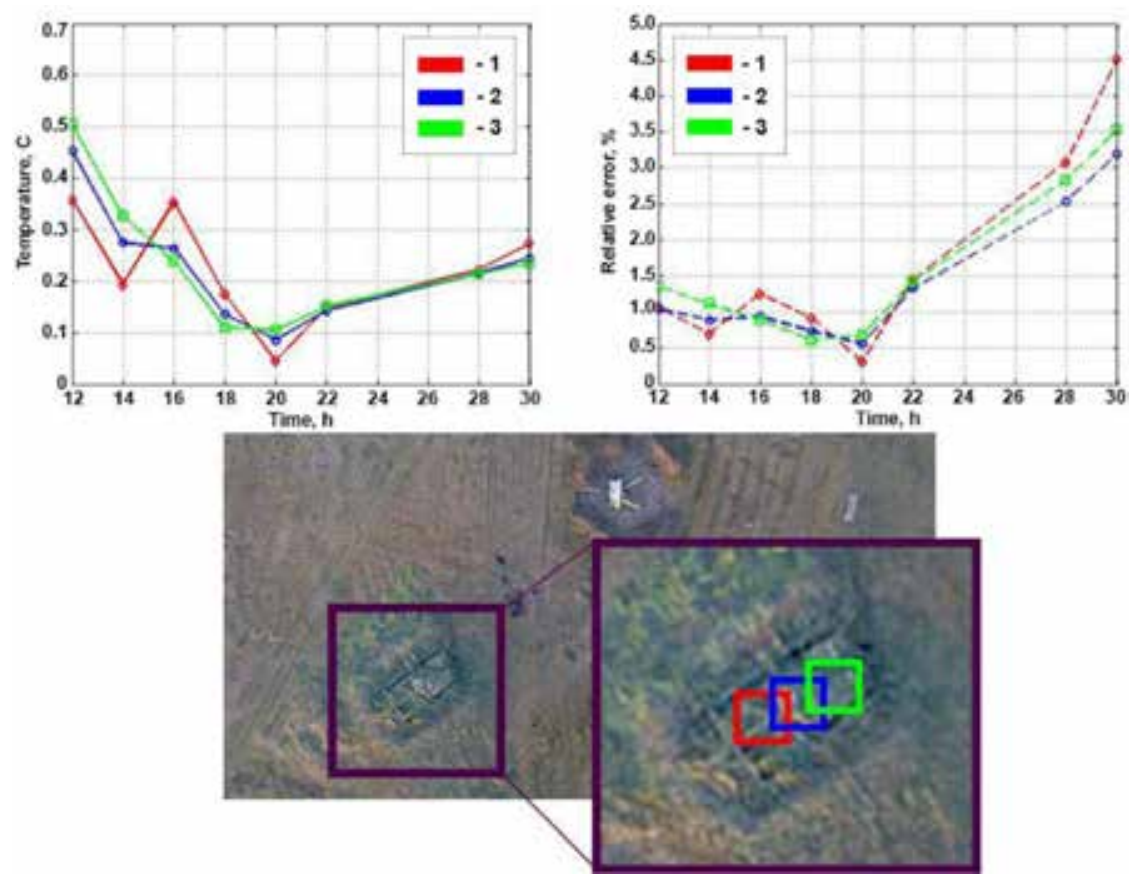

Рис. 8. Динамика изменения усредненной невязки по температуре (слева) и относительной погрешности (справа) моделирования температурных полей объекта «макет танка под маскировочным комплектом МКТ-2» во времени: 1 - пиксель с координатами $\mathrm{x}=118 ; \mathrm{y}=444 ; 2$ - пиксель с координатами $\mathrm{x}=130$; $\mathrm{y}=437 ; 3-$ пиксель с координатами $\mathrm{x}=138 ; \mathrm{y}=431$; площадь участка для всех точек $\Delta \mathrm{x}=15$ пикс.; $\Delta y=15$ пикс.

Fig. 8. Dynamics of changes in the average of the residual temperature (left) and relative error (right) simulation of the temperature fields of the object "layout of the tank under the camouflage kit MKT-2" in time: 1 - pixel with coordinates $\mathrm{x}=118 ; \mathrm{y}=444 ; 2-$ pixel with coordinates $\mathrm{x}=130 ; \mathrm{y}=437 ; 3$ - pixel with coordinates $\mathrm{x}=138$; $\mathrm{y}=431$; plot size for all points, $\Delta \mathrm{x}=15$ pixels; $\Delta \mathrm{y}=15$ pixels

1. Получать распределения температурных полей «объектов-аналогов» и подстилающих поверхностей районов ДМ по тепловым томограммам в зависимости от их географического положения и метеорологических условий для заданного времени суток.

2. Прогнозировать инфракрасные сигнатуры «объектов-аналогов» и подстилающих поверхностей районов ДМ в зависимости от времени суток, географического положения, а также метеорологических условий.

3. Получать исходные данные (в виде пространственного распределения температурных полей) районов ДМ для дальнейшей оценки динамики изменения вероятностных характеристик распознавания (различения) «объектов-аналогов» на фоне подстилающих поверхностей и определения временных интервалов для наиболее эффективного ведения ДМ при заданных географическом положении и метеорологических условиях.

\section{Благодарности / Acknowledgements}

Работа выполнена при финансовой поддержке РФФИ (грант № 18-08-00053А).

The work was supported by the RFBR grant no. 18-08-00053A. 


\section{Список литературы / References}

[1] Ищук И.Н., Филимонов А.М., Постнов К.В., Степанов Е.А., Дмитриев Д.Д. Корреляционная обработка кубоида инфракрасных изображений, получаемых с беспилотных летательных аппаратов. Ч. 2. Метод обработки инфракрасных сигнатур эталонных объектов на основе численного решения нелинейной задачи теплообмена. Журнал Сибирского федерального университета. Техника и технологии, 2016, 9(3), 376-384 [Ishchuk I.N., Filimonov A.M., Postnov K.V., Stepanov E.A., Dmitriev D.D. Correlation processing of cuboid infrared images obtained from unmanned aerial vehicles. Part 2. Method of processing infrared signatures of reference objects on the basis of numerical solution of nonlinear heat transfer problem, Journal of Siberian Federal University, Engineering and technologies, 2016, 9(3), 376-384 (in Russian)].

[2] Ищук И.Н., Долгов А.А., Бебенин А.А., Панов С.А. Расчет пространственного распределения температурных полей при дистанционном мониторинге поверхности территорий с беспилотного летательного аппарата. Журнал Сибирского федерального университета. Техника и технологии, 2018, 11(3), 273-279 [Ishchuk I.N., Dolgov A.A., Bebenin A.A., Panov S.A. Calculation of spatial distribution of temperature fields at remote monitoring of the territory surface from unmanned aerial vehicle, Journal of Siberian Federal University, Technics and technologies, 2018, 11(3), 273-279 (in Russian)].

[3] Ищук И.Н., Долгов А.А. Способ классификации техногенных объектов на основе построения многослойных тепловых томограмм, Сборник трудов ИТНТ-2019 по материалам V международной конференции и молодежной школы «Информационные технологии и нанотехнологии», 21-24 мая 2019 г., Самара, 2019, 610-619 [Ischuk I.N., Dolgov A.A. The method of classification of technogenic objects on the basis of multilayer thermal tomography, Proceedings of ITNT-2019 according to the materials of the V international conference and young scientists school "Information technology and nanotechnology", may 21-24, 2019, Samara, 2019, 610-619 (in Russian)].

[4] Šimůnek J. The HYDRUS-1D Software Package for Simulating the One-Dimensional Movement of Water, Heat, and Multiple Solutes in Variably-Saturated Media.Version 3.0/J.Šimuinek, M.Th. van Genuchten, M. Šejna. California: Preprint Department of environmental sciences university of California riverside, 2005. P. 270.

[5] Ищук И.Н., Филимонов А.М., Долгов А.А., Степанов Е.А., Тяпкин В.Н. Алгоритм совместной обработки многоспектральных изображений по данным воздушной съемки с беспилотных летательных аппаратов, Промылиленные АСУ и контроллеры, 2018, 10, $27-34$ [Ishchuk I.N., Filimonov A.M., Dolgov A.A., Stepanov E.A., Tyapkin V.N. Algorithm of joint processing of multispectral images according to aerial survey data from unmanned aerial vehicles, Industrial ACS and controllers, 2018, 10, $27-34$ (in Russian)].

[6] Архив погоды в Воронеже [Электронный ресурс] - Режим доступа: http://rp5.ru/Архив_погоды_в _Воронеже - Заглавие с экрана. [Weather archive in Voronezh [Electronic resource] - Access: http://rp5.ru/Apхив_погоды_в_Воронеже].

[7] Ищук И.Н., Долгов А.А., Филимонов А.М., Дмитриев Д.Д. Методика оценки динамики изменения вероятностных характеристик распознавания объектов воздушной разведки. Журнал Сибирского федерального университета. Техника и технологии, 2019, 12(6), 683-693 [Ishchuk I.N., Dolgov A.A., Filimonov A.M., Dmitriev D.D. Technique of estimation of dynamics of change of probabilistic characteristics of recognition of objects of aerial reconnaissance, Journal of the Siberian Federal University, Technics and technologies, 2019, 12(6), 683-693 (in Russian)]. 